\title{
Random Access Policies for Wireless Networked Control Systems with Energy Harvesting Sensors
}

\author{
Miguel Calvo-Fullana, Carles Antón-Haro, Javier Matamoros, and Alejandro Ribeiro
}

\begin{abstract}
In this paper, we study wireless networked control systems with energy harvesting sensors. Multiple sensors share a wireless medium over which they transmit measurements to their respective controllers, and due to the shared medium, packet collisions occur if sensors transmit simultaneously. To alleviate this problem, we propose random access policies that satisfy a desired control performance on each control loop, while also satisfying the energy constraints imposed by the energy harvesting process. The optimal scheduling policy is shown to follow a simple thresholding operation. Moreover, we provide a stochastic dual method for their computation, which is shown to be decoupled across sensors. Finally, we verify numerically the properties of the proposed policy.
\end{abstract}

\section{INTRODUCTION}

Wireless networked control systems are rapidly becoming prevalent in the modern world. They are present in smart homes, robotic automation, smart transportation, industrial plants and more. A critical component of these wireless control systems are the sensing devices. These sensor nodes measure the state of the system and transmit their observations over a wireless channel. However, due to the uncertain nature of the wireless channel, the choice of communication policy critically affects the closed loop performance of the control system. The sensors share the wireless communication medium and therefore one should aim for an efficient use of this resource in a way that meets the control performance requirements.

Also, due to the small and of low power consumption of sensor nodes, they have recently become a prime candidate to be powered by energy harvesting technologies. This refers to the capability of sensors to acquire energy from environmental sources (such as solar or wind energy [1]), and thus increasing their potential lifetime. This has led to a great deal of research interest in EH-powered communication, with problems ranging from throughput maximization [2][4], source-channel coding [5]-[7], estimation [8]-[10] and others being studied (see [11] for a comprehensive overview).

When dealing with classically powered sensors, the scheduling problem in wireless networked control systems

This work was partially supported by the Catalan government under grant SGR2014-1567; the Spanish government under grant TEC2013-44591-P (INTENSYV) and grant PCIN-2013-027 (E-CROPS) in the framework of the ERA-NET CHIST-ERA program; and the European Commission under grant FP7-607774 (ADVANTAGE).

M. Calvo-Fullana, C. Antón-Haro, and J. Matamoros are with the Centre Tecnològic de Telecomunicacions de Catalunya (CTTC/CERCA), 08860 Castelldefels, Barcelona, Spain (e-mail: miguel.calvo@cttc.cat; javier.matamoros@cttc.cat; carles.anton@cttc.cat).

A. Ribeiro is with the Department of Electrical and Systems Engineering, University of Pennsylvania, Philadelphia, PA 19104 USA (e-mail: aribeiro@ seas.upenn.edu).

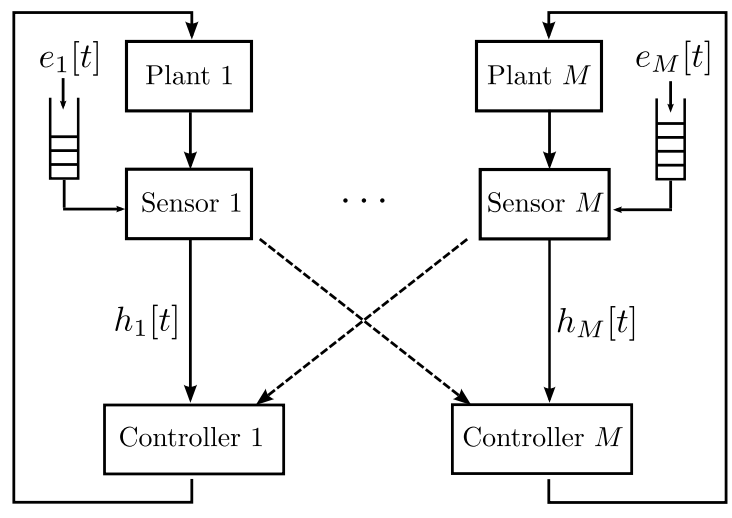

Fig. 1. System model.

has been previously studied in the literature. The most common approach to this problem is the design of centralized scheduling policies. In this setup, there exists an overseeing entity specifying which sensor is allowed to transmit at a given time slot, in order to avoid collisions between packets. These type of policies might be static [12], [13] or of a more dynamic nature, where centralized decisions can be taken based on plant state information [14] or wireless channel conditions [15], among others.

In this work, we study the design of random access policies for sensor nodes powered by energy harvesting. Different from works [13]-[15], we design decentralized scheduling policies. This is more in line with the policy presented in [16], which we extend here to take into account the energy harvesting process. Our goal is to design channel access policies such that all control loops satisfy their control performance requirements and the power consumption satisfies the energy causality constraints imposed by the energy harvesting process. To this end, we use a control performance abstraction which allows us to translate the control performance requirements to successful transmission probabilities of the random access scheme. Under ergodic assumptions on the channel states and energy harvesting process, we propose a simple dynamic threshold scheduling policy which accounts for the channel as well as the battery state of the sensor. Furthermore, the optimal scheduling policies are computed by means of a stochastic dual method. Finally, we numerically verify the behavior of the proposed policies.

\section{System Model and Problem Formulation}

Consider the wireless control system consisting of $M$ different plants shown in Figure 1. The state of each of these 
plants is measured by a sensor powered by energy harvesting, and each of these sensors transmits the measurements to the plant controller through a shared wireless medium. Due to the nature of the shared medium, collisions will arise if more than one sensor decides to transmit at the same time. Under these conditions, our goal is to design medium access policies such that a specific control performance is guaranteed for all plants.

\section{A. Control model}

Let us denote by $\gamma_{i}[t] \in\{0,1\}$ whether the transmission by the $i$-th sensor at time slot $t$ was successful or not. Then, under the assumption of a linear time-invariant system, we can model the plant dynamics by the following discrete-time switched system

$$
x_{i}[t+1]= \begin{cases}A_{c, i} x_{i}[t]+w_{i}[t], & \text { if } \gamma_{i}[t]=1, \\ A_{o, i} x_{i}[t]+w_{i}[t], & \text { if } \gamma_{i}[t]=0,\end{cases}
$$

where $x_{i}[t] \in \mathbb{R}^{n_{i}}$ denotes the $i$-th plant state at time slot $t$, and the $w_{i}[t]$ variables correspond to independent and identically distributed (i.i.d.) zero-mean Gaussian noise with covariance $C_{i}$. Moreover, $A_{c, i} \in \mathbb{R}^{n_{i} \times n_{i}}$ is the closedloop dynamics matrix and $A_{o, i} \in \mathbb{R}^{n_{i} \times n_{i}}$ is the open-loop dynamics matrix. The closed-loop matrix $A_{c, i}$ corresponds to the system evolution when the plant successfully receives the measurement transmitted by the sensor node and is thus assumed to be asymptotically stable. On the other hand, the matrix $A_{o, i}$ corresponds to the plant evolution when not receiving the sensor measurement (due to a lack of transmission by the sensor node or collision) and may be unstable.

\section{B. Communication model}

Consider now the communication model. We have defined the variables $\gamma_{i}[t]$ to indicate the successful transmission of the sensor nodes measurements. Hence, they correspond to random variables whose distribution depends on the chosen communication policy. We consider a time slotted communication model in which at each time slot, the sensor either transmits a packet (containing the measurement at that time slot) over the shared wireless medium or not. We denote this decision to transmit by the scheduling variable $z_{i}[t] \in\{0,1\}$. Then, if two sensors transmit at the same time slot, a collision will occur and neither packet will be received. Furthermore, the packet will be decoded with a certain probability depending on the channel state. Then, the probability of successful transmission at a given time slot $t$ is given by

$$
\operatorname{Pr}\left(\gamma_{i}[t]=1\right)=q\left(h_{i}[t]\right) z_{i}[t] \prod_{j \neq i}\left(1-z_{j}[t]\right)
$$

where $q: \mathbb{R} \rightarrow[0,1]$ is a continuous strictly increasing decoding function, which depends on the channel state $h_{i}[t]$ (A typical decoding function is shown in Figure 2). Furthermore, the channel state variables $h_{i}[t]$ are assumed

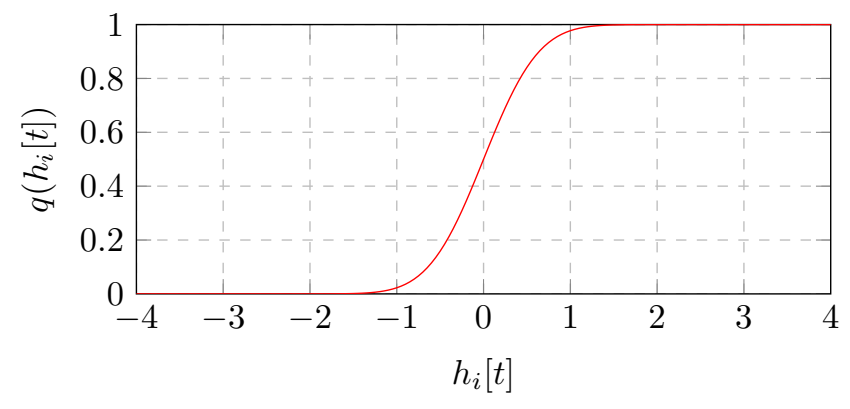

Fig. 2. Probability of decoding as a function of the channel state.

to have continuous probability density function ${ }^{1}$. Also, for notational compactness we define $q_{i}[t] \triangleq q\left(h_{i}[t]\right)$.

\section{Energy harvesting}

We consider the sensor nodes to be powered by energy harvesting. At time slot $t$, the $i$-th sensor harvests $e_{i}[t]$ units of energy, where the energy harvesting process is assumed to be stationary. This energy is then stored in a battery of capacity $b_{i}^{\max }$. Furthermore, we assume a normalized power consumption model, where accessing the medium consumes one unit of energy. By considering this transmission cost as the only energy-consuming action taken by the sensor nodes, we can model the battery dynamics as

$$
b_{i}[t+1]=\left[b_{i}[t]-z_{i}[t]+e_{i}[t]\right]_{0}^{b_{i}^{\max }}
$$

where $[\cdot]_{0}^{b_{i}^{\max }}$ denotes the projection to the interval $\left[0, b_{i}^{\max }\right]$. Also, in order to ensure that the sensors do not consume more energy than they have available, the following energy causality constraint must be satisfied for all time slots

$$
z_{i}[t] \leq b_{i}[t] .
$$

\section{Control performance}

We are interested in the design of communication policies that satisfy a desired control performance criteria. Due to the nature of the communication system, we can establish a relationship between the sensor transmission probabilities and the control performance as stated by the following proposition.

Proposition 1 (Control performance abstraction [16]). Consider the switched system described by (1) with $\gamma_{i}[t]$ given by a sequence of i.i.d. Bernoulli random variables, and the quadratic Lyapunov function $V_{i}\left(x_{i}\right)=x_{i}^{T} P_{i} x_{i}$, with $P_{i}$ positive definite. Then the function $V_{i}\left(x_{i}\right)$ decreases at an average rate $\rho_{i}<1$, denoted by

$$
\mathbb{E}\left[V_{i}\left(x_{i}[t+1]\right) \mid x_{i}[t]\right] \leq \rho_{i} V\left(x_{i}[t]\right)+\operatorname{tr}\left(P_{i} C_{i}\right)
$$

if and only if $\operatorname{Pr}\left(\gamma_{i}[t]=1\right) \geq p_{i}$, where $p_{i}$ is given by

$p_{i}=\min \left\{\theta \geq 0: \theta A_{c, i}^{T} P_{i} A_{c, i}+(1-\theta) A_{o, i}^{T} P_{i} A_{o, i} \leq \rho_{i} P_{i}\right\}$

\footnotetext{
${ }^{1}$ This assumption holds true for many practical channel models such as Rician, Nakagami or Rayleigh fading [17].
} 
Proof. By particularizing the function $V_{i}\left(x_{i}\right)=x_{i}^{T} P_{i} x_{i}$ with the system dynamics (1), we can write the equation

$$
\begin{aligned}
\mathbb{E}\left[V_{i}\left(x_{i}[t+1]\right) \mid x_{i}[t]\right] & =x_{i}^{T}[t] A_{c, i}^{T} P_{i} A_{c, i} x_{i}[t] \operatorname{Pr}\left(\gamma_{i}[t]=1\right) \\
& +x_{i}^{T}[t] A_{o, i}^{T} P_{i} A_{o, i} x_{i}[t] \operatorname{Pr}\left(\gamma_{i}[t]=0\right) \\
& +\operatorname{tr}\left(P_{i} C_{i}\right) .
\end{aligned}
$$

Then, by substituting this expression in the left hand side of the average decrease inequality (5) we have the following inequality

$$
\begin{aligned}
& x_{i}^{T}[t] A_{c, i}^{T} P_{i} A_{c, i} x_{i}[t] \operatorname{Pr}\left(\gamma_{i}[t]=1\right) \\
& \quad+x_{i}^{T}[t] A_{o, i}^{T} P_{i} A_{o, i} x_{i}[t] \operatorname{Pr}\left(\gamma_{i}[t]=0\right) \leq \rho_{i} x_{i}^{T}[t] P_{i} x_{i}[t] .
\end{aligned}
$$

Since this condition needs to hold for all $x_{i}[t]$, we can equivalently rewrite this condition as the following linear matrix inequality

$$
\begin{aligned}
A_{c, i}^{T} P_{i} A_{c, i} & \operatorname{Pr}\left(\gamma_{i}[t]=1\right) \\
& +A_{o, i}^{T} P_{i} A_{o, i}\left(1-\operatorname{Pr}\left(\gamma_{i}[t]=1\right)\right) \leq \rho_{i} P_{i},
\end{aligned}
$$

where we have also used the fact that $\operatorname{Pr}\left(\gamma_{i}[t]=0\right)=(1-$ $\left.\operatorname{Pr}\left(\gamma_{i}[t]=1\right)\right)$. Then, the $\operatorname{Pr}\left(\gamma_{i}[t]=1\right)$ values satisfying this inequality define a convex set of which there is a minimum value $p_{i}$ such that the condition is equivalent to $\operatorname{Pr}\left(\gamma_{i}[t]=\right.$ 1) $\geq p_{i}$.

\section{E. Problem Formulation}

The previous proposition allows us to relate the desired control performance to the required packet success rates in the communication scheme. Our goal is then to design communication policies such that this control performance is attained, while also satisfying the energy causality constraints (4). Assuming an ergodic mode of operation, the control performance of the system is determined by the long term behavior of the transmission probabilities (2). Hence, the following ergodic limits determine the control performance

$$
p_{i} \leq \lim _{t \rightarrow \infty} \frac{1}{t} \sum_{l=1}^{t} q_{i}[l] z_{i}[l] \prod_{j \neq i}\left(1-z_{j}[l]\right)
$$

Likewise, the energy causality constraints (4) have a long term behavior determined by the ergodic limits

$$
\lim _{t \rightarrow \infty} \frac{1}{t} \sum_{l=1}^{t} z_{i}[l] \leq \lim _{t \rightarrow \infty} \frac{1}{t} \sum_{l=1}^{t} e_{i}[l]
$$

where, in this case the energy causality constraints (4) are satisfied in an ergodic sense. Nonetheless, we will provide conditions ensuring that they are satisfied in a time slot to time slot basis. Furthermore, in order to make an efficient use of the shared medium, we aim to find policies satisfying (10) and (11) that minimize the number of times the medium is accessed. Due to the ergodic assumption on the system, we can substitute the ergodic limits in (10) and (11) for their expected value. This allows us to pose the following stochastic optimization problem

$$
\begin{array}{cl}
\underset{z_{i} \in \mathcal{Z}}{\operatorname{minimize}} & \sum_{i=1}^{M} \mathbb{E} z_{i} \\
\text { subject to } & p_{i} \leq \mathbb{E} q_{i} z_{i} \prod_{j \neq i}\left(1-z_{j}\right), i=1, \ldots, M \\
& \mathbb{E} z_{i} \leq \mathbb{E} e_{i} \quad i=1, \ldots, M
\end{array}
$$

where the expectation is taken with respect to the channel realizations $h_{i}[t]$ and the energy harvesting process $e_{i}[t]$, and $\mathcal{Z}$ is the set of all functions $\mathbb{R}^{+} \rightarrow\{0,1\}$. We desire that the sensors be able to solve this problem in a distributed manner. However, the optimization problem (12) depends on the other sensors scheduling decision by constraint (12b). In order to separate the problem in a per sensor basis we take the logarithm of constraint (12b) as follows

$$
\log \left(p_{i}\right) \leq \log \left(\mathbb{E} q_{i} z_{i}\right)+\sum_{j \neq i} \log \left(1-\mathbb{E} z_{j}\right),
$$

and introduce the auxiliary variables $r_{i}=\mathbb{E} q_{i} z_{i}$ and $s_{i}=$ $\mathbb{E} z_{i}$. This allows us to rewrite problem (12) in the following equivalent form

$$
\begin{aligned}
\underset{z_{i} \in \mathcal{Z}, r, y \in[0,1]}{\operatorname{minimize}} & \sum_{i=1}^{M} \mathbb{E} z_{i} \\
\text { subject to } & \log \left(p_{i}\right) \leq \log \left(r_{i}\right)+\sum_{j \neq i} \log \left(1-s_{j}\right) \\
& \quad i=1, \ldots, M \\
& r_{i} \leq \mathbb{E} q_{i} z_{i}, \quad i=1, \ldots, M \\
& s_{i} \geq \mathbb{E} z_{i}, \quad i=1, \ldots, M \\
& \mathbb{E} z_{i} \leq \mathbb{E} e_{i} \quad i=1, \ldots, M
\end{aligned}
$$

where the relaxation of the auxiliary variables to an inequality is done without loss of optimality. The optimal solution of (14) is equivalent to the optimal solution of (12). Nonetheless, another problem arises due to the fact that the sensors do not have knowledge of the distributions of $h_{i}$ nor the energy harvesting process $e_{i}$ (over which the expectation is taken). Nonetheless, they have access to the instantaneous values $h_{i}[t]$ and $e_{i}[t]$. Thus, by leveraging stochastic subgradient ascent on the dual domain [18], we will design an algorithm that solves problem (14) while satisfying constraints (4) for all time slots.

\section{Stochastic Algorithm}

Let us define the vector $z=\left\{z_{i}, r_{i}, s_{i}\right\}$ collecting the primal variables and the vector $\lambda=\left\{\phi_{i}, \nu_{i}, \mu_{i}, \beta_{i}\right\}$ collecting the dual variables. We can write the Lagrangian of problem (14) as follows 


$$
\begin{aligned}
\mathcal{L}(z, \lambda) & =\sum_{i=1}^{M} \mathbb{E} z_{i} \\
& +\sum_{i=1}^{M} \phi_{i}\left(\log \left(p_{i}\right)-\log \left(r_{i}\right)-\sum_{j \neq i} \log \left(1-s_{j}\right)\right) \\
& +\sum_{i=1}^{M} \nu_{i}\left(r_{i}-\mathbb{E} q_{i} z_{i}\right)+\sum_{i=1}^{M} \mu_{i}\left(\mathbb{E} z_{i}-s_{i}\right) \\
& +\sum_{i=1}^{M} \beta_{i}\left(\mathbb{E} z_{i}-\mathbb{E} e_{i}\right)
\end{aligned}
$$

And the Lagrange dual function is then given by

$$
g(\lambda)=\max _{z} \mathcal{L}(z, \lambda) .
$$

By approximating the expected values for the instanteneous values and reordering the Lagrangian (15), the values of the scheduling variables can be found as the solution to the minimization

$$
z_{i}[t]=\underset{z_{i} \in\{0,1\}}{\arg \min } z_{i}\left(1-\nu_{i}[t] q_{i}[t]+\mu_{i}[t]+\beta_{i}[t]\right) .
$$

Since the scheduling variable $z_{i}[t]$ acts linearly on the term $1-\nu_{i}[t] q_{i}[t]+\mu_{i}[t]+\beta_{i}[t]$, the solution to this minimization problem corresponds to the simple threshold rule given by $z_{i}[t]=\mathbb{I}\left(\nu_{i}[t] q_{i}[t]-\mu_{i}[t]-\beta_{i}[t] \geq 1\right)$, where $\mathbb{I}(\cdot)$ denotes the indicator function. In a similar manner, the auxiliary variables are given by the minimizations

$$
\begin{gathered}
r_{i}[t]=\underset{r_{i} \in[0,1]}{\arg \min }-\phi_{i}[t] \log \left(r_{i}\right)+\nu_{i}[t] r_{i} \\
s_{i}[t]=\underset{s_{i} \in[0,1]}{\arg \min }-\sum_{i=1}^{M} \phi_{i}[t] \sum_{j \neq i} \log \left(1-s_{j}\right)-\sum_{i=1}^{M} \mu_{i}[t] s_{i}
\end{gathered}
$$

which have the following closed form solutions

$$
r_{i}[t]=\left[\frac{\phi_{i}[t]}{\nu_{i}[t]}\right]_{0}^{1}, \quad s_{i}[t]=\left[1-\frac{\sum_{j \neq i} \phi_{i}[t]}{\mu_{i}[t]}\right]_{0}^{1},
$$

where $[\cdot]_{0}^{1}$ denotes the projection to the interval $[0,1]$. Note that the computation of the auxiliary variable $s_{i}[t]$ requires the dual variables $\phi_{i}[t]$ of the other sensors. This implies that a certain amount of communication overhead needs to occur to ensure this information exchange takes place. Following, since the dual function (16) is concave, we can perform a subgradient ascent on the dual domain. The updates to the dual variables are then given by

$$
\begin{aligned}
\phi_{i}[t+1]=\left[\phi_{i}[t]+\log \left(p_{i}\right)\right. & -\log \left(r_{i}[t]\right) \\
& \left.-\sum_{j \neq i} \log \left(1-s_{i}[t]\right)\right]^{+}
\end{aligned}
$$

$\overline{\text { Algorithm } 1 \text { Control-aware scheduling algorithm for energy }}$ harvesting sensors.

1: Initialize: Initialize the dual variables to $\phi_{i}[0]:=0$, $\nu_{i}[0]:=0, \mu_{i}[0]:=0$, and $\beta_{i}[0]:=b_{i}^{\max }-b_{i}[0]$.

2: Step 1: The sensor decides to access the shared medium according to

3: $z_{i}[t]:=\mathbb{I}\left(\nu_{i}[t] q_{i}[t]-\mu_{i}[t]-\beta_{i}[t] \geq 1\right)$

4: Step 2: The sensor computes the auxiliary variables variables

5: $r_{i}[t]:=\left[\frac{\phi_{i}[t]}{\nu_{i}[t]}\right]_{0}^{1}$ and $s_{i}[t]:=\left[1-\frac{\sum_{j \neq i} \phi_{i}[t]}{\mu_{i}[t]}\right]_{0}^{1}$

6: Step 3: The sensor updates the dual variables

\begin{tabular}{l} 
7: $\phi_{i}[t+1]:=\left[\phi_{i}[t]+\log \left(p_{i}\right)-\log \left(r_{i}[t]\right)\right.$ \\
$\left.-\sum_{j \neq i} \log \left(1-s_{i}[t]\right)\right]$ \\
8: $\nu_{i}[t+1]:=\left[\nu_{i}[t]+r_{i}[t]-z_{i}[t] q_{i}[t]\right]_{0}^{\nu^{\max }}$ \\
9: $\mu_{i}[t+1]:=\left[\mu_{i}[t]+z_{i}[t]-s_{i}[t]\right]^{+}$ \\
10: $\beta_{i}[t+1]:=\left[\beta_{i}[t]+z_{i}[t]-e_{i}[t]\right]_{0}^{b_{i}^{\max }}$ \\
11: Step 4: Set $t:=t+1$ and go to Step 1. \\
\hline
\end{tabular}

$$
\begin{gathered}
\nu_{i}[t+1]=\left[\nu_{i}[t]+r_{i}[t]-z_{i}[t] q_{i}[t]\right]_{0}^{\nu_{i}^{\max }} \\
\mu_{i}[t+1]=\left[\mu_{i}[t]+z_{i}[t]-s_{i}[t]\right]^{+} \\
\beta_{i}[t+1]=\left[\beta_{i}[t]+z_{i}[t]-e_{i}[t]\right]_{0}^{b_{\max }}
\end{gathered}
$$

where for simplicity we have used an unit step size and projected the dual variables to a specific interval. Let us denote by $\nu_{i}^{\star}$ and $\beta_{i}^{\star}$ the optimal Lagrange multipliers of $\nu_{i}$ and $\beta_{i}$, respectively. Then if $\nu_{i}^{\star} \in\left[0, \nu_{i}^{\max }\right]$ and $\beta_{i}^{\star} \in\left[0, \beta_{i}^{\max }\right]$, the convergence of Algorithm 1, which summarizes the proposed policy, can be shown.

Also, note that due to the use of a unit step size, the dual update (24) mimics the battery dynamics (3) in a mirrored way, since they can be expressed as $b_{i}[t]=b_{i}^{\max }-\beta_{i}[t]$. This observation leads us to the following proposition, by which we can choose a battery capacity such that the energy causality constraints (4) are satisfied for all time slots.

Proposition 2 (Energy Causality). Let the battery capacity of the $i$-th sensor satisfy $b_{i}^{\max } \geq \nu_{i}^{\max }-1$. Then Algorithm 1 satisfies the energy causality constraints $z_{i}[t] \leq b_{i}[t]$ for all $t$ time slots.

Proof. Assume the battery capacity satisfies $b_{i}^{\max } \geq \nu_{i}^{\max }$ 1. Then, to satisfy the energy causality constraints $z_{i}[t] \leq$ $b_{i}[t]$ for all $t$, it suffices to certify that no transmission is scheduled when the sensor has no stored energy, i.e., $z_{i}[t]=$ 0 when $b_{i}[t]=0$. When $b_{i}[t]=0$, the battery multiplier $\beta_{i}[t]$ takes the value $\beta_{i}[t]=b_{i}^{\max }$. And, by (17), we have that the sensor transmits if $\nu_{i}[t] q_{i}[t]-\mu_{i}[t]-\beta_{i}[t] \geq 1$. Hence, the sensor transmits if $\nu_{i}[t] q_{i}[t]-\mu_{i}[t]-b_{i}^{\max } \geq 1$. Furthermore, since $q_{i}[t] \leq 1$ and $\nu_{i}[t]$ is bounded by the projection to $\nu_{i}^{\max }$, we can certify that no transmission occurs if $\nu_{i}^{\max }-b_{i}^{\max } \leq 1$. This is ensured by the battery capacity assumption $b_{i}^{\max } \geq \nu_{i}^{\max }-1$. 


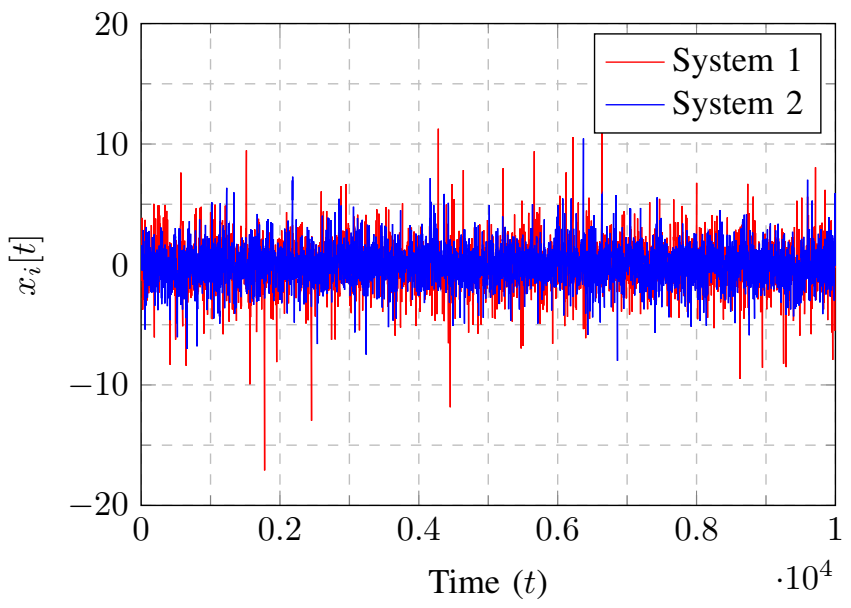

Fig. 3. Evolution of the plant state at each time slot.

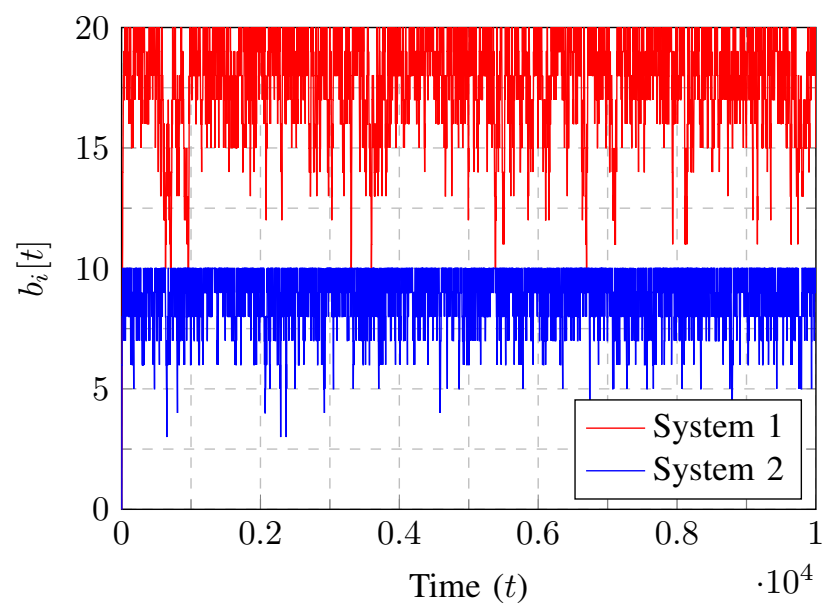

Fig. 4. Energy stored in the batteries at each time slot.

\section{NuMERICAL RESUlts}

In this section we study the performance of the proposed random access scheme with energy harvesting sensors. We consider a scalar control system, with $M=2$ plants sharing the communication medium. The plant dynamics are given by $A_{o, 1}=1.15$ and $A_{c, 1}=0.1$ for the first system, and $A_{o, 2}=1.05$ and $A_{c, 2}=0.15$ for the second system. Hence, the first system is slightly more unstable than the second one. Further, we consider both systems to be perturbed by i.i.d. zero-mean Gaussian noise. We assume the same control performance requirement for both plants, given by the Lyapunov function $V_{i}\left(x_{i}[t]\right)=x_{i}^{2}$ and an expected decrease rate of $\rho_{i}=0.8$. By Proposition 1 this translates to successful transmission probabilities of $p_{1} \approx 0.3981$ and $p_{2} \approx 0.2801$, respectively.

Further, we consider the sensors in both systems to be powered by an energy harvesting process of rate $e_{i}=1$. The sensors store this energy in batteries of size $b_{1}^{\max }=20$ and $b_{2}^{\max }=10$ for the first and second sensors, respectively. Since the first system is slightly more unstable, it requires a larger battery capacity as we will see later. Furthermore,

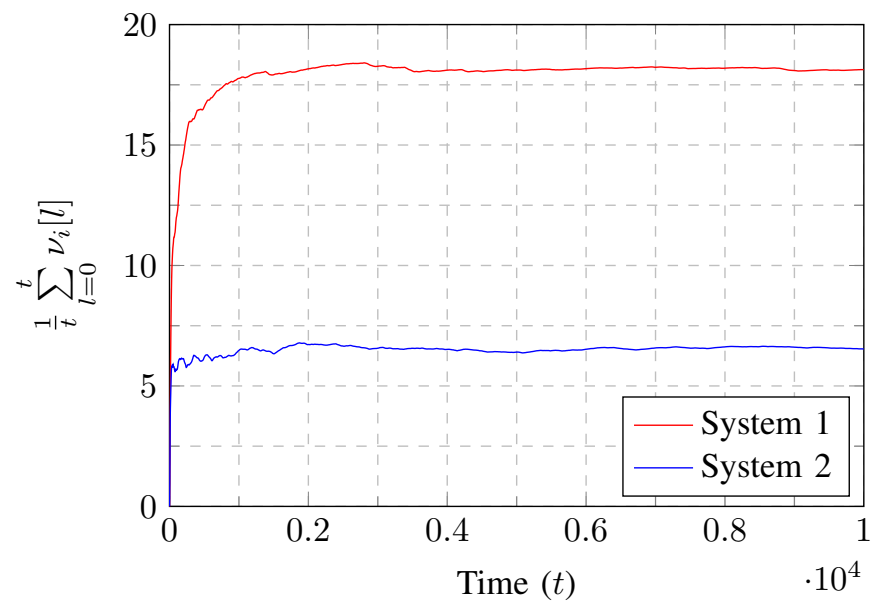

Fig. 5. Average evolution of the dual variables $\nu_{i}[t]$ at each time slot.

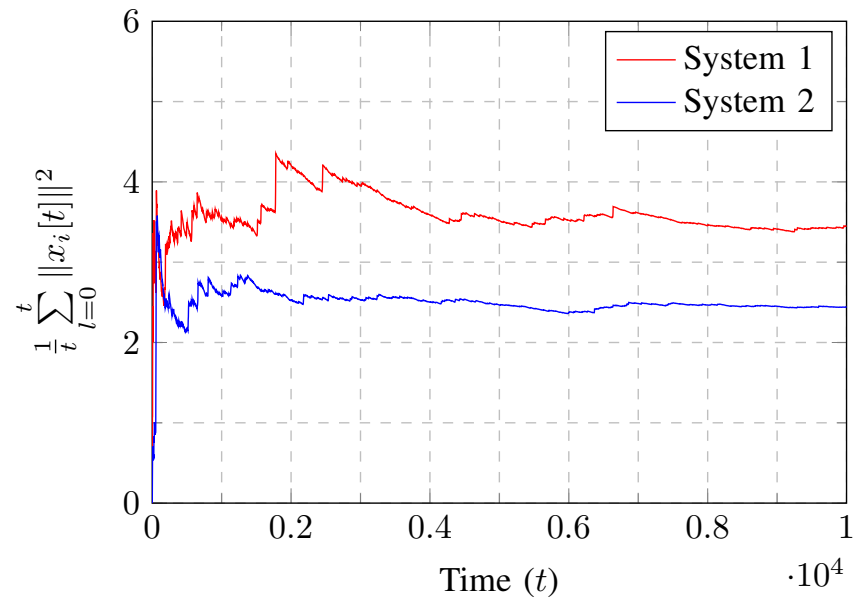

Fig. 6. Evolution of the control system performance at each time slot.

the projection of the dual variables $\nu_{i}$ is chosen to be $b_{i}^{\max }=\nu_{i}^{\max }$ for both systems, hence satisfying Proposition 2. Finally, we consider a communication system where the channel variables $h_{i}[t]$ are i.i.d. zero-mean Gaussian variables and the decoding probability $q\left(h_{i}[t]\right)$ is given by the function shown in Figure 2.

In Figure 3 we plot the plant state at each time slot. As previously noted, the first system is slightly more unstable than the second system, as can be evidenced by the more pronounced peaks of instability. This instability translates to a higher variance of the energy stored in the batteries, as evidenced by Figure 4. Here, we see that the energy states vary more drastically in the more unstable system. Also, the battery size chosen for the second system is smaller.

In Figure 5 we plot the average value $\frac{1}{t} \sum_{l=0}^{t} \nu_{i}[l]$ of the $\nu_{i}$ dual variables. The average values of the $\nu_{i}$ multipliers converge to the values $\nu_{1}^{\star} \approx 18.2$ and $\nu_{2}^{\star} \approx 6.5$, which by Proposition 2 evidences the lower battery requirements of System 2. Finally, we show in Figure 6 the evolution of the control system performance, where we see that both systems are asymptotically stable. 


\section{CONClusions}

In this work, we have provided a random access mechanism for energy harvesting sensors in wireless networked control systems. The goal has been to design communication policies such that a desired control performance is attained while also satisfying the energy causality requirements imposed by the energy harvesting process. Under ergodic assumptions, we have proposed a decentralized scheduling policy and provided a stochastic dual-based algorithm to compute it. Numerical results have verified the stabilizing properties of the proposed scheme.

\section{REFERENCES}

[1] R. J. Vullers, R. Schaijk, H. J. Visser, J. Penders, and C. V. Hoof, "Energy harvesting for autonomous wireless sensor networks," IEEE Solid-State Circuits Magazine, vol. 2, no. 2, pp. 29-38, 2010.

[2] J. Yang and S. Ulukus, "Optimal packet scheduling in an energy harvesting communication system," IEEE Transactions on Соттиnications, vol. 60, no. 1, pp. 220-230, 2012.

[3] K. Tutuncuoglu and A. Yener, "Optimum transmission policies for battery limited energy harvesting nodes," IEEE Transactions on Wireless Communications, vol. 11, no. 3, pp. 1180-1189, 2012.

[4] O. Ozel, K. Tutuncuoglu, J. Yang, S. Ulukus, and A. Yener, "Transmission with energy harvesting nodes in fading wireless channels: Optimal policies," IEEE Journal on Selected Areas in Communications, vol. 29, no. 8, pp. 1732-1743, 2011.

[5] M. Calvo-Fullana, J. Matamoros, and C. Antón-Haro, "Reconstruction of correlated sources with energy harvesting constraints in delayconstrained and delay-tolerant communication scenarios," IEEE Transactions on Wireless Communications, 2017.

[6] O. Orhan, D. Gunduz, and E. Erkip, "Source-channel coding under energy, delay, and buffer constraints," IEEE Transactions on Wireless Communications, vol. 14, no. 7, pp. 3836-3849, July 2015.

[7] P. Castiglione, O. Simeone, E. Erkip, and T. Zemen, "Energy management policies for energy-neutral source-channel coding," IEEE Transactions on Communications, vol. 60, no. 9, pp. 2668-2678, 2012.

[8] M. Calvo-Fullana, J. Matamoros, and C. Antón-Haro, "Sensor selection and power allocation strategies for energy harvesting wireless sensor networks," IEEE Journal on Selected Areas in Communications, vol. 34, no. 12, pp. 3685-3695, 2016.

[9] J. Matamoros, M. Calvo-Fullana, and C. Antón-Haro, "On the impact of correlated sampling processes in wsns with energy-neutral operation," in 2015 IEEE International Conference on Communications (ICC). IEEE, 2015, pp. 258-263.

[10] M. Calvo-Fullana, J. Matamoros, C. Antón-Haro, and S. M. Fosson, "Sparsity-promoting sensor selection with energy harvesting constraints," in Acoustics, Speech and Signal Processing (ICASSP). 2016 IEEE International Conference on, March 2016.

[11] S. Ulukus, A. Yener, E. Erkip, O. Simeone, M. Zorzi, P. Grover, and K. Huang, "Energy harvesting wireless communications: A review of recent advances," IEEE Journal on Selected Areas in Communications, vol. PP, no. 99, pp. 1-1, 2015.

[12] W. Zhang, M. S. Branicky, and S. M. Phillips, "Stability of networked control systems," IEEE Control Systems, vol. 21, no. 1, pp. 84-99, 2001.

[13] D. Hristu-Varsakelis, "Feedback control systems as users of a shared network: Communication sequences that guarantee stability," in Decision and Control, 2001. Proceedings of the 40th IEEE Conference on, vol. 4. IEEE, 2001, pp. 3631-3636.

[14] G. C. Walsh, H. Ye, and L. G. Bushnell, "Stability analysis of networked control systems," IEEE transactions on control systems technology, vol. 10, no. 3, pp. 438-446, 2002.

[15] K. Gatsis, M. Pajic, A. Ribeiro, and G. J. Pappas, "Opportunistic control over shared wireless channels," IEEE Transactions on Automatic Control, vol. 60, no. 12, pp. 3140-3155, 2015.

[16] K. Gatsis, A. Ribeiro, and G. J. Pappas, "Random access design for wireless control systems," arXiv preprint arXiv:1605.00627, 2016.

[17] A. Goldsmith, Wireless communications. Cambridge University Press, 2005.
[18] A. Ribeiro, "Ergodic stochastic optimization algorithms for wireless communication and networking," IEEE Transactions on Signal Processing, vol. 58, no. 12, pp. 6369-6386, 2010. 